\title{
THE USE OF 3D VIRTUAL PLANNING AND 3D PRINTING FOR THE TREATMENT OF FACIALASYMMETRY:A CASE REPORT
}

\author{
Eman Shaheen ${ }^{1,2 a^{*}}$, Constantinus Politis ${ }^{1,2 b}$ \\ 'Department of Imaging \& Pathology, Faculty of Medicine, KU Leuven, Belgium \\ 2Department of Oral and Maxillofacial Surgery, University Hospitals of Leuven, Belgium \\ ${ }^{\mathrm{B} B S c}, \mathrm{MSC}$, PhD, Clinical Engineer \\ ${ }^{b} \mathrm{MD}, \mathrm{DDS}, \mathrm{MHA}, \mathrm{MM}, \mathrm{PhD}$, Professor and Head of Department of Oral and Maxillofacial
}

Cite this article:

Shaheen E, Politis C. The use of 3D virtual planning and 3D printing for the treatment of facial asymmetry: a case report. Stoma Edu J.2016;3(3-4):156-161.

Introduction: Facial asymmetry is a three-dimensional problem and is common in humans. Depending on the severity of the condition and the cause, several treatment modalities are proposed. In this study, we report on our workflow treating facial mandibular asymmetries without malocclusion.

Methodology: CT scans were used for preoperative planning and postoperative evaluation. 3D planning using the mirroring technique was presented and afterwards a simulated extension was 3D printed and used during the surgery for accurate harvesting and placement of the bone graft.

Results: The surgical actual outcome when compared to the simulation revealed good approximation with a mean of $0.29( \pm 1.73) \mathrm{mm}$.

Conclusion: The proposed method can be considered promising for the treatment of facial mandibular asymmetry without affecting the occlusion. However, a larger scale prospective study should be conducted to confirm these findings.

Keywords: facial asymmetry, 3D virtual planning, 3D printing.

\section{Introduction}

People with facial asymmetry are commonly seen in the general population. The developmental type of facial asymmetry is idiopathic and nonsyndromic in nature ${ }^{1}$. Facial asymmetry may cause esthetic, malocclusion or other problems to the patients.

Different treatment modalities of facial asymmetry have been proposed including orthodontic appliances for mild asymmetry in children ${ }^{2}$. For more severe asymmetries, involving dental, skeletal and soft tissue components, a combination of orthodontic and orthognathic management are recommended. Orthognathic surgery can be accompanied by mandibular angle reduction, genioplasty, bony augmentation, etc ${ }^{1}$.

The aim of this study was to present the use of 3D virtual planning and printing as surgical assistance to the treatment of facial mandibular asymmetry with autogenous appositional bone graft.

\section{Materials and Methods}

A patient was presented with complaints of facial asymmetry to the department of Oral and maxillofacial surgery, University hospitals of Leuven, Belgium. The patient had no complaints regarding functional occlusion, only esthetic. After clinical and radiographic examination, the patient was diagnosed with neutral occlusion within normal skeletal and dental midlines, good transverse ratio, end-to-end in the front with a chin deviation to the left.

The procedure recommended for this patient was treatment of mandibular asymmetry with autogenous bone graft. Indication for this patient was purely esthetic.

Ethical approval was obtained from the Ethical Review Board of the University Hospitals Leuven (S57587).

\section{*Corresponding author:}

Eman Shaheen, BSc, MSc, PhD, Clinical Engineer, Responsible of the 3D Surgical Orthognathic Lab, Department of Oral and Maxillofacial Surgery,

University Hospitals Leuven, Campus Sint-Rafaël, Kapucijnenvoer 33, BE-3000 Leuven, Belgium

Tel/Fax: +32 16341854; e-mail: eman.shaheen@uzleuven.be 


\subsection{Preoperative planning}

Preoperative imaging was performed with a 128-slice spiral multi-slice computer tomography (MSCT) scanner (Siemens Somatom Definition Flash, Germany: 120 kV, 161 mAS, slice thickness $0.75 \mathrm{~mm}$ ). The Digital Imaging and Communications in Medicine (DICOM) images of the patient were imported into the Simplant O\&O software (Materialise Dental, Leuven, Belgium) and the mandible, maxilla, skull and soft tissue were segmented via thresholding (Fig. 1a).

A mirroring procedure of the mandible was implemented around the midsagittal plane as defined by Gateno et al. (3)(Fiq. 1b). The left side was mirrored to the right and the right was mirrored to the left (Fig. 1c and 1d). As shown in figure 1, the mirroring from left to right wasn't feasible since the mandibular alveolar nerve would be exposed, therefore, only the mirroring of the right to the left was considered. This option concerns an augmentation procedure (Fig. 1c). Afterwards the original mandible was subtracted from the mirrored mandible at the left side (Fig. 1 e) and exported as STereoLithography (STL) file then 3D printed in polymer material using Objet Connex 350 (Stratasys, Eden Prairie, Minnesota, USA) with slice thickness of $0.03 \mathrm{~mm}$.

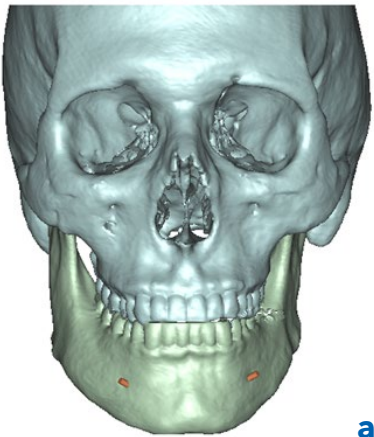

a.

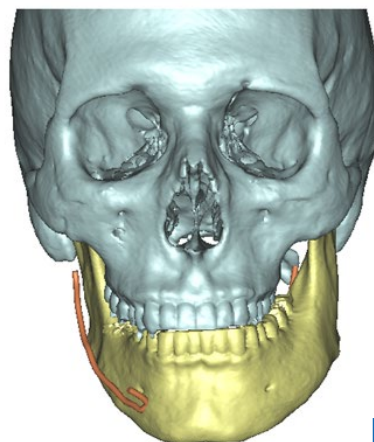

b.

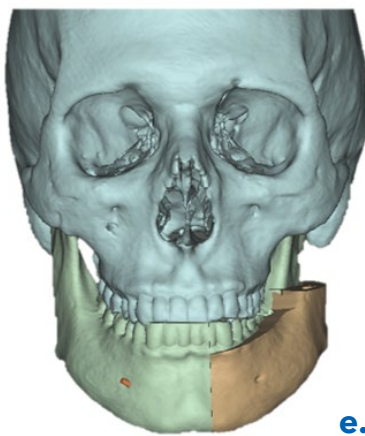

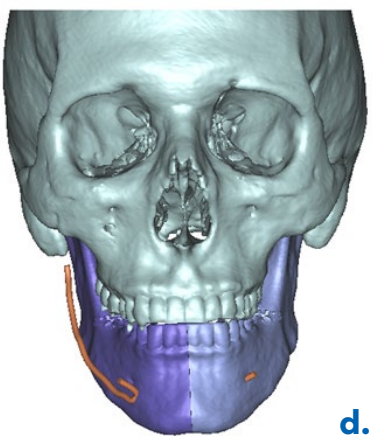

d.

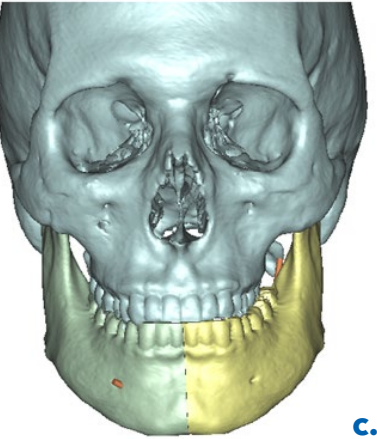

c. e.

Figure 1. a. Preoperative skull. b. Preoperative skull with mirrored mandible. c. Preoperative skull with mirrored mandible right to left. $d$. Preoperative skull with mirrored mandible left to right.

e. Preoperative skull with mirrored mandible right to left subtracted
This subtracted object served as a surgical guide for the bone graft size and shape during the operation (Fig. 2). This object was sterilized prior to the operation.

The results of the mirroring and the expected soft tissue simulation were shown to the patient prior to the operation (Fig. $7 \mathrm{a}, \mathrm{b}$ ). The patient accepted these simulations and the fact that the postoperative results may differ from the simulations.
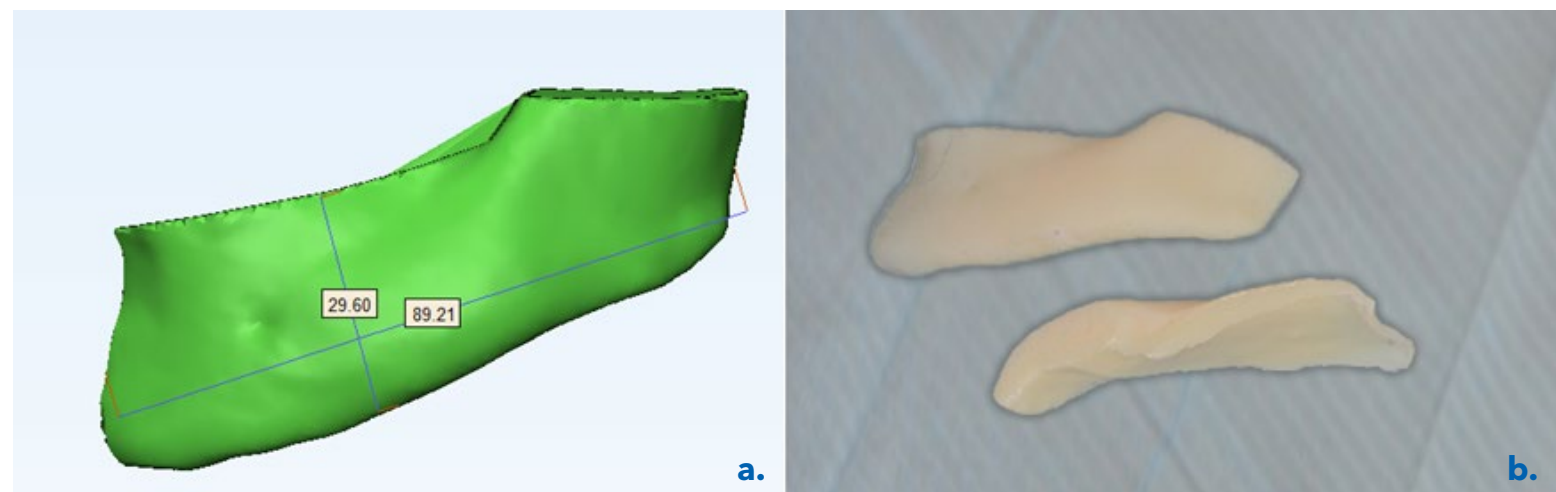

Figure 2. a. The surgical guide in STL format (dimensions: $29.6 \mathrm{~mm} \times 89.2 \mathrm{~mm}$ ). b. The same object in the operation room after 3D printing and sterilization 


\subsection{Operation}

The 3D printed surgical template was used during the bone harvesting of the anterior iliac crest where a rectangular piece of bone larger than the template was harvested, using the template to define the bone cuts. Thereafter the singlepiece bone graft was manually trimmed using the template as target model, which was afterwards grafted on the body of the left side of the mandible. The surgeon built up the mandible with the bone and fixated the different pieces with 6 mini screws
(KLS Martin, Germany) of lengths 7 and $9 \mathrm{~mm}$.

\subsection{Postoperative evaluation}

Postoperative CT was taken one month after the operation with the same settings as for the preoperative scan. The data was evaluated in the Amira software (FEI, USA). The preoperative and postoperative DICOM images were imported and the postoperative images were superimposed on the preoperative images via voxel based registration with mutual information ${ }^{4-6}$ (Fig. 3).
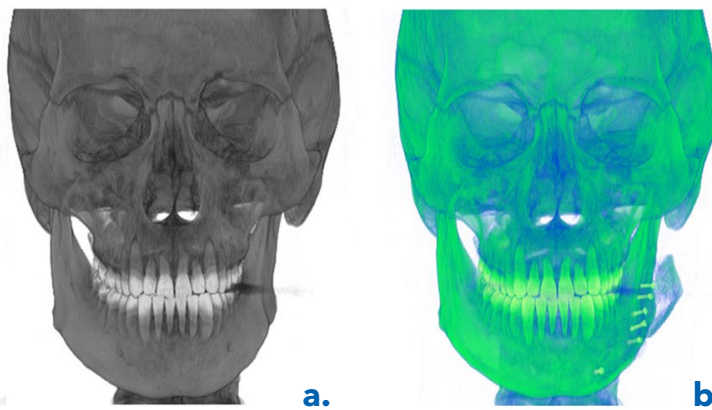

b.

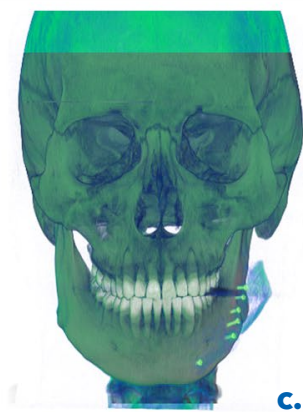

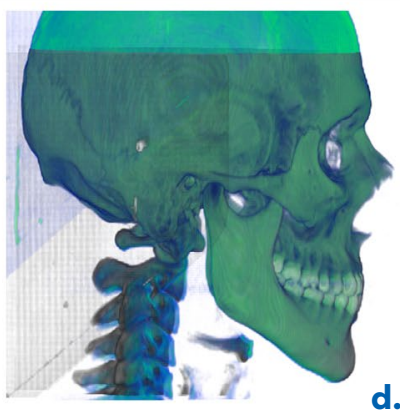

Figure 3. a. Preoperative skull (gray). b. Postoperative skull (green). c. Postoperative skull registered onto the preoperative skull frontal view, and $d$. lateral view

The preoperative extension was imported into the Amira project to visualize the changes between the simulated extension and the postoperative outcome (Fig. 4).

The postoperative bone was segmented and the extension was separately segmented then exported as STL files for further analysis.
A part comparison analysis between the postoperative extension and the simulated extension was done in the 3-matic software (Materialise, Leuven, Belgium) where the Euclidean distances between the surfaces were calculated (Fig. 5).
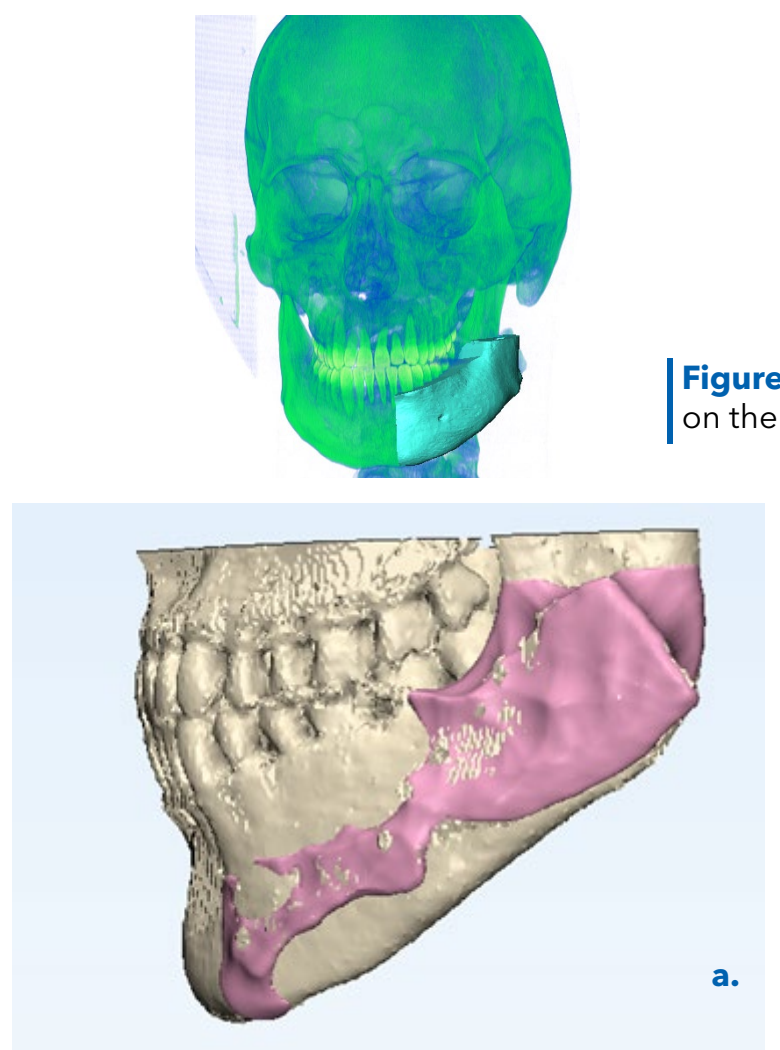

a.

Figure 4. The simulated preoperative extension overlaid on the postoperative outcome after registration

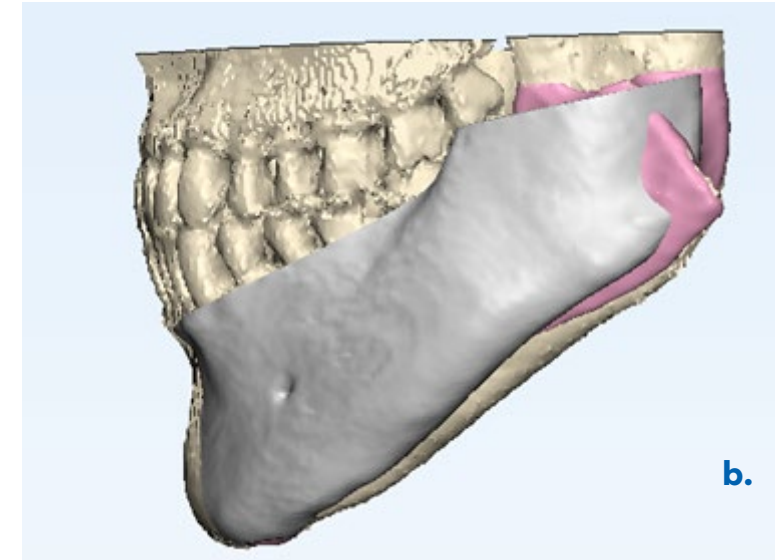

Figure 5. a. The postoperative mandible with the postoperative segmented extension.

b. The postoperative mandible and extension with the preoperative simulated extension 


\section{Results}

The visual evaluation of the simulated and actual extensions revealed a good approximate in terms of shape and position. For the quantitative analysis, at the bone level via the part comparison, revealed a mean of $0.29 \mathrm{~mm}$ and standard deviation of 1.73 $\mathrm{mm}$. Fig. 6 shows the results of the part comparison with the simulated extension overlaid on the postoperative extension and the color coded map ranging in $\mathrm{mm}$. Extreme differences $(5 \mathrm{~mm})$ were found at the region of mandibular angle while differences at the mandibular body region ranged between 0 and $3 \mathrm{~mm}$.

Fig. 8 shows the extra-oral clinical images taken for this patient preoperatively (left) and 6 months postoperatively (right).

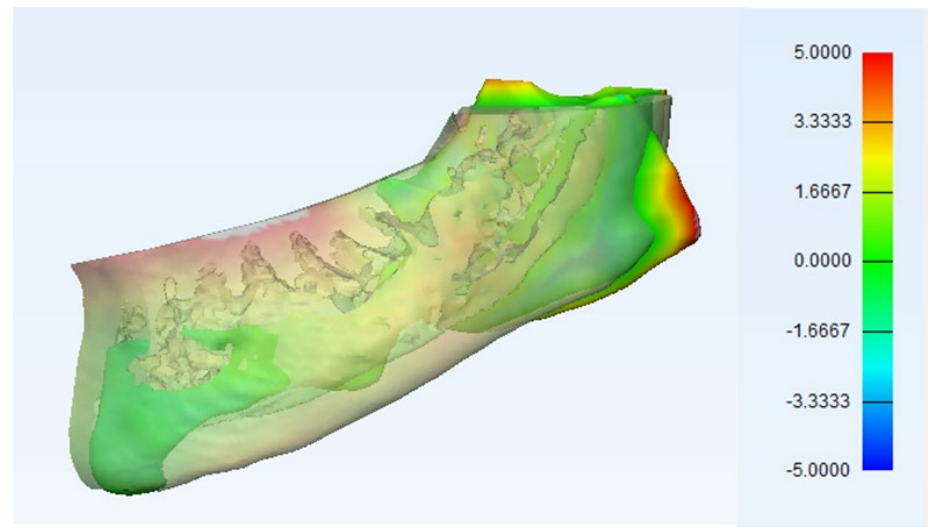

Figure 6. Color coded map for the part comparison analysis between the simulated and actual extensions

\section{Discussion}

In this study we presented a case with facial mandibular asymmetry. As the patient's complaints and diagnosis were only esthetic with no functional problems, the recommended treatment was autogenous apposition bone graft. Even though other non-resorbable graft materials could equally be used by the same method, the patient opted for an autogenous graft material. The use of 3D planning and printing was investigated where the mirroring technique around the midsagittal plane was applied to simulate the symmetry of the mandible both sides. A 3D printed extension was used during the surgery as a guide for harvesting

and grafting for accurate positioning.

For the quantitative postoperative evaluation for the bone in relation to the simulation revealed mean error of $0.29 \mathrm{~mm}( \pm 1.73 \mathrm{~mm})$. Maximum differences were found at the mandibular angle part. This can be due to the difficulty of reshaping the bone in this region.

Since it is recommended to evaluate the soft tissue minimum 6 months postoperatively (7), quantitative evaluation one month postoperatively was not possible, but visually it was in line with the preoperative prediction (Fig. 7).
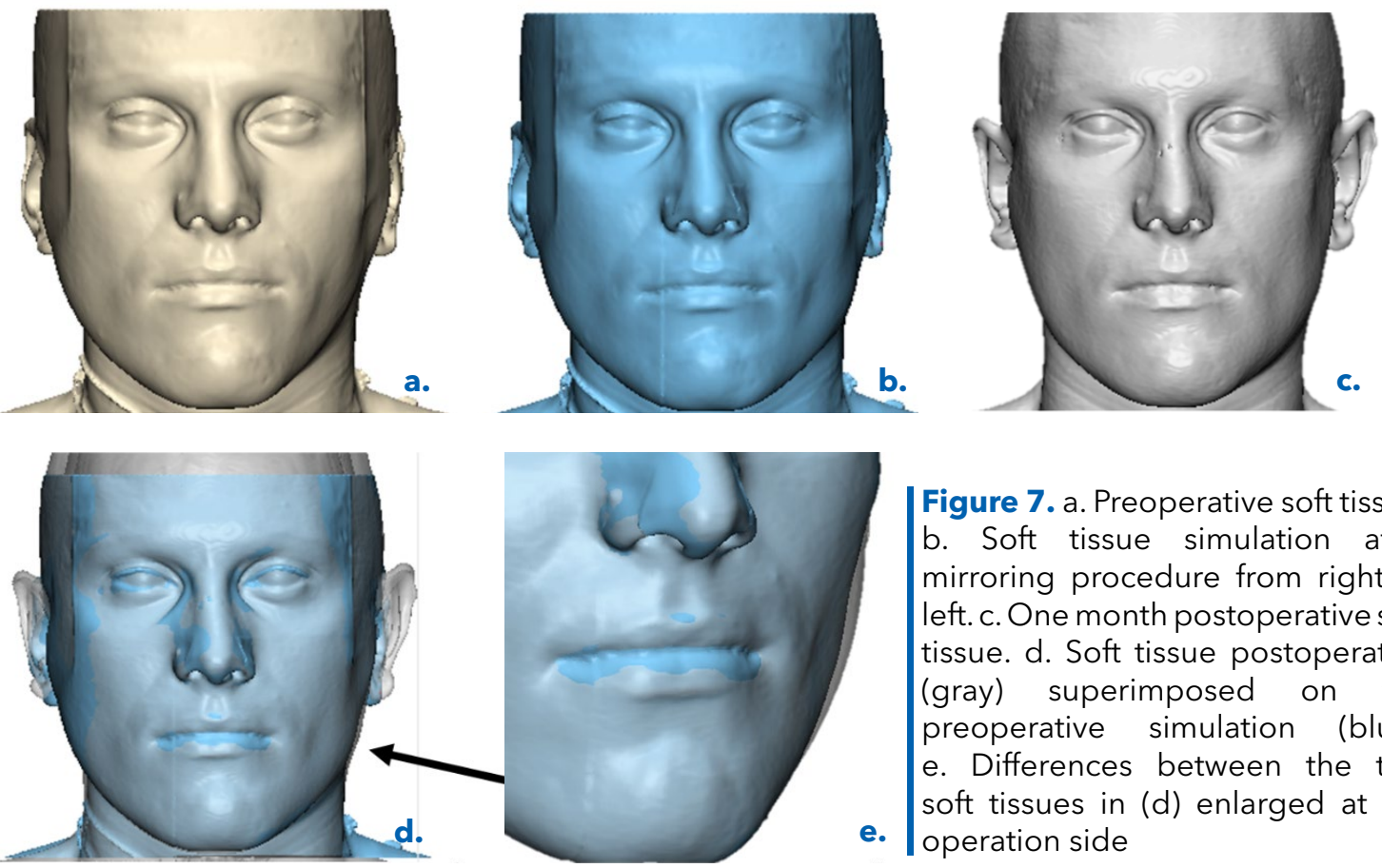

Figure 7. a. Preoperative soft tissue. b. Soft tissue simulation after mirroring procedure from right to left.c. One month postoperative soft tissue. d. Soft tissue postoperative (gray) superimposed on the preoperative simulation (blue). e. Differences between the two soft tissues in (d) enlarged at the e. operation side 
This limitation can be solved in future studies by taking regularly $3 \mathrm{D}$ photographs and thus no extra radiation dose to the patient ${ }^{8}$. However, after clinical evaluation of the patient 6 months

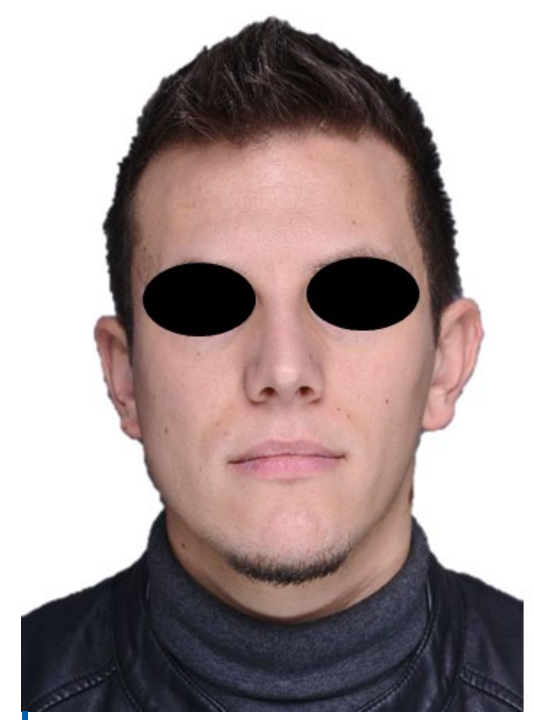

Figure 5. The extra oral non-smiling clinical images of the patient preoperatively (left) and 6 months postoperatively (right)

\section{Conclusions}

Based on the findings presented in this case, the treatment of mandibular asymmetry using autogenous appositional bone graft can be recommended. The use of 3D planning based on the mirroring technique and the $3 \mathrm{D}$ printing of the extension was proven to be valuable for more accurate harvesting and grafting during the surgery as shown in the results. However, a larger scale prospective study should be conducted to support these outcomes. postoperative, the surgical outcome was clinically accepted and the patient was satisfied with the results (Fig. 8).

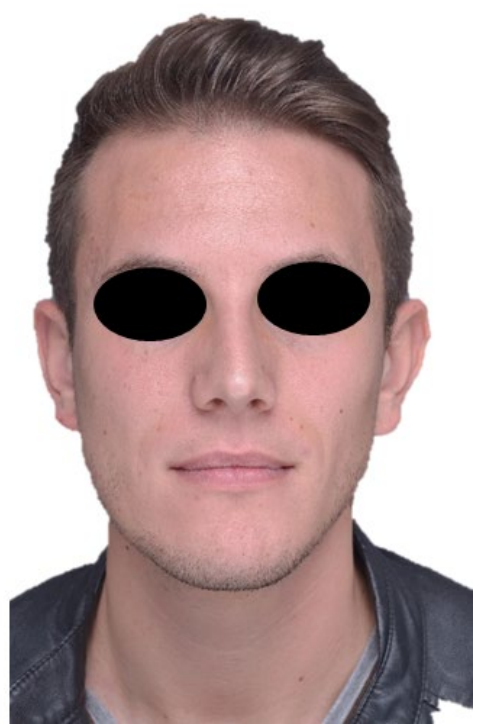

\section{Authors Contributions}

ES and CP contributed equally to the concept and protocol. ES contributed to the data gathering and analysis. ES and CP contributed to the interpretation and critical manuscript writing and revision.

\section{Acknowledgments}

The authors declares no conflict of interest related to this study. There are no conflicts of interest and no financial interests to be disclosed.

\section{REFERENCES}

1. Cheong YW, Lo LJ. Facial asymmetry: etiology, evaluation, and management. Chang Gung Med J. 2011;34(1):341-351.

2. Converse JM, Horowitz SL, Coccaro PJ, Wood-Smith D.The corrective treatment of the skeletal asymmetry in hemifacial microsomia. Plast Reconstr Surg. 1973;52(3):221-231.

3. Gateno J, Xia JJ, Teichgraeber JF. New 3-dimensional cephalometric analysis for orthognathic surgery. J Oral Maxillofac Surg. 2011;69(3):606-622. doi.org/10.1016/j.joms.2010.09.010 4. Viola $P$, Chao A. Multiple sensor image alignment by maximization of mutual information. In: Massachusetts Institute of Technology and ALPHATECH, Inc. 1995.

5. Pluim JP, Maintz JB, Viergever MA. Mutual information based registration of medical images: a survey. IEEE Trans Med Imaging. 2003;22(8):986-1004.
6. Collignon A, Maes F, Delaere D, Vandermeulen D, Suetens $P$ Marchal G. Automated multi-modality image registration based on information theory. In: Bizais Y, Barillot C, Di Paola R. (eds) Information Processing in Medical Imaging. Dordrecht, The Netherlands: Kluwer Press Publishers; 1995;263-274.

7. Bailey LJ, Collie FM, White RP Jr. Long-term soft tissue changes after orthognathic surgery. Int J Adult Orthodon Orthognath Surg. 1996;11(1):7-18.

8. Hajeer MY, Ayoub AF, Millett DT. Three-dimensional assessment of facial soft-tissue asymmetry before and after orthognathic surgery. Br J Oral Maxillofac Surg. 2004;42(5):396-404. 


\section{Eman SHAHEEN}

BSc, MSc, PhD, Clinical Engineer

Department of Imaging \& Pathology

Faculty of Medicine, KU Leuven, Belgium

Eman Shaheen graduated from the faculty of Computer Sciences (2003), Cairo University, Egypt where she also worked as a teaching assistant till 2007. She obtained her doctoral degree in 2014, KU Leuven, Belgium in Medical Imaging with focus on 3D simulations in breast tomosynthesis. In the same year, she started working in the Department of Maxillo-Facial Surgery, University Hospitals Leuven-Belgium as a Clinical Engineer. Her main responsibilities are 3D planning of orthognathic surgeries, orthodontic and other dental specialities. Next to the patient related work, she is part of the OMFSIMPATH research group (KU Leuven, Belgium) where she supervises students, supports different research projects related to 3D printing and 3D simulations. Her main project of interest is to improve the CMF protocol for orthognathic surgeries next to other research related projects.

\section{Questions}

\section{Which software was used for the mirroring technique?}
口a. Amira;
b. Simplant O\&O;
口c. 3-matic;
d. Proplan.

\section{Which type of registration was used to superimpose the postoperative images to the preoperative images?}

$\square$ a. Point based registration;

b. Surface based registration;

ac. Voxel based registration;

$\square$ d. Surface best fit.

\section{What type of analysis was used to compare the simulated and postoperative extension?}

$\square$ a. Part comparison surface based;

b. Part comparison volumetric based;

c. Point to point comparison;

$\square$ d. Linear measurements.

\section{What were the complaints of the patient?}

$\square$ a. Esthetic;

b. Malocclusion;

c. Esthetic and malocclusion;

口d. Skeletal class II. 